\title{
Notizen vom Hermann-Heller-Symposion in Berlin
}

Am 5. November 1933 starb Hermann Heller (geboren am 17. Juli 1891) im spanischen Exil. Zum Gedenken an ihn fand vom 4.-6. November 1983 in Berlin ein von Christoph Müller (FU Berlin) und Ilse Staff (Frankfurt) organisiertes Symposion mit Unterstützung der Stifung Volkswagenwerk statt. 32 Wissenschaftler hatten dazu vorher Beiträge verfaßt, die Gegenstand der Gespräche der größtenteils anwesenden Verfasser und einiger weiterer Gäste auf dem Symposion waren. Der folgende Bericht versteht sich daher zugleich als (notwendig subjektive und unvollständige) Zusammenfassung der Diskussionen des Symposions und als Ankündigung der Veröffentlichung der Beiträge in einer Gedächtnisschrift ${ }^{1}$. Bericht und Ankündigung lassen sich vorweg dahin zusammenfassen, daß das Symposion mit den etwa gleichzeitig erschienenen Schriften über Heller ${ }^{2}$ eine zweite Welle der Heller-Rezeption nach der ersten durch das Buch W. Schluchters, die Biographie K. Meyers und die Ausgabe der Gesammelten Schriften gekennzeichneten ${ }^{3}$ darstellt.

I. Das Symposion begann im ominös benannten Otto Braun-Saal der Preußischen Staatsbibliothek mit einer öffentlichen Festsitzung vor internationalem Publikum, auf das die Brillanz einer Eröffnungsrede des scheidenden und insofern offenbar persönlich betroffenen Berliner Universitätspräsidenten Eberhard Lämmert strahlte. Lämmert zeichnete den Gegensatz zwischen idealistisch-autonomer und gesellschaftsbezogener Wissenschaft und die Tradition des Verdikts gegen die »Politisierung « nach, die einem Hermann Heller im Richtungsstreit so schwer zu schaffen machen mußte. War die Beschreibung der Frontstellung Hellers gegen Kelsen noch eher von traditionellen Erklärungsmustern bestimmt, so war die Verortung der Differenz von Heller und Radbruch als Vorwegnahme des Positivismusstreits höchst anregend, die Differenz von Heller und Carl Schmitt im Hinblick auf die Normalität als Ziel einfach zutreffend. Wenn Lämmert Heller insofern als wichtigen Wegweiser bezeichnete, so diagnostizierte er damit aktuelle Scheidewege.

Wolfgang Abendroth (Frankfurt) arbeitete in seinem Eröffnungsvortrag über die

I Christoph Müller/Ilse Staff (Hrsg.), Der soziale Rechtsstaat. Gedächtnisschrift für Hermann Heller. 1891-1933, Baden-Baden 1984. - Es sei betont, daß die folgenden Ausführungen auf den Diskussionsnotizen des Verfassers beruhen, für die Zusammenfassung der (viel umfangreicheren) Diskussionen komprimiert und systematisiert wurden, und daß sie den Diskussionrednern vor Drucklegung nicht zur Genehmigung vorgelegt werden konnten. Mißverständnisse und die Formulierung im einzelnen verantwortet deshalb der Verfasser.

2 Abgesehen von der Neuauflage von Hermann Heller, Staatslehre, 6. Aufl. Tübingen, r983, und der grundlegenden Schrift Wolfgang Schluchters, Entscheidung für den sozialen Rechtsstaat. Hermann Heller und die staatstheoretische Diskussion in der Weimarer Republik, 2. Aufl. Baden-Baden 1983, sind hervorzuheben Stephan Albrecht, Hermann Hellers Staats- und Demokratieauffassung, Frankfurt 1 983 ; Gerhard Robbers, Hermann Heller: Staat und Kultur, Baden-Baden 1983. Rudolf Waser, Die sozialistische Idee im Denken Hermann Hellers, Diss.Basel 1982, liegt vorerst nur als Dissertationsdruck vor.

3 Wolfgang Schluchter (zit. Anm. 2), I. Aufl. Köln 1968; Klaus Meyer, Hermann Heller. Eine biographische Skizze, Politische Vierteljahresschrift 8, 1967, S. 293; Hermann Heller, Gesammelte Schriften, 3 Bde., Leiden/Tübingen 1971 . 
Bedeutung Hellers für die Weimarer Republik dessen Sonderstellung heraus. Wenn die andern bedeutenderen jungen Staatsrechtslehrer der zwanziger Jahre gegen Kelsen polemisierten, dann aus einer sich dem Faschismus nähernden Gegnerschaft gegen den Positivismus. Heller dagegen erkannte spätestens nach seiner Italienreise die Gefahr des Faschismus und prangerte sie an. Während die anderen Gegner des Positivismus sich nach 1933 anpaßten, soweit sie nicht Deutschland verlassen mußten, weil sie Juden waren, war Heller außer aus rassischen auch aus wissenschaftlichen und politischen Gründen im nationalsozialistischen Staat nicht tragbar. Dies führte dazu, daß die in Deutschland Gebliebenen staatsrechtliche Schulen begründen konnten, während Hellers Einfluß in der Bundesrepublik gering, sein Schüler Drath im Bundesverfassungsgericht isoliert blieb. Schon in der Weimarer Zeit gab es nur wenige demokratisch gesonnene Staatsrechtslehrer. Für die Jüngeren war daher $\mathrm{Hel}-$ lers Bedeutung als Vorbild groß, zumal Kelsen für eine politische Orientierung weniger hilfreich erschien. Allerdings hat auch Heller nicht gegen die Reichsexekution in Thüringen und Sachsen 1923 Stellung bezogen, und gegen den Preußenschlag nahm er nur vor Gericht Stellung, während ein realer Kampf nötig gewesen wäre. Insofern gingen die Jüngeren schon damals über seine Position hinaus; aber sie konnten, da sie noch nicht auf Lehrstühle gelangt waren, vor 1933 keinen größeren Einfluß erlangen.

Konkreteren Fragen des aktuellen Verfassungsrechts wandte sich Ilse Staff in einem Vortrag zur Aktualität der Staatstheorie Hellers, speziell zur Frage des sozialen Rechtsstaats zu. Ausgehend von Hellers Begriffsmerkmalen des sozialen Rechtsstaats insistierte sie auf dem Postulat, der demokratische Prozeß müsse eine inhaltliche Auseinandersetzung mit der Gestaltung der Wirtschaftsordnung führen. Dem stellte sie das besonders seit I967 (Art. I09 II GG) die Wirtschaftspolitik der Bundesrepublik beherrschende Gleichgewichtsdenken gegenüber, das die verfassungsrechtliche Zuständigkeitsordnung, vor allem die Macht des Parlaments, leerlaufen läßt. Wenn sich Sozialstaatlichkeit nur noch auf dem Weg der Konjunktursteuerung aktualisiert, wird die Verteilungsproblematik entpolitisiert. Grundrechte werden entweder zum juristischen Verteilungsproblem oder zu gesellschaftspolitisch irrelevanten Ausgrenzungen. Das Management der Verteilung bleibt der Regierung überlassen, ohne daß sich Interessen der Bürger ihr gegenüber demokratisch artikulieren. Soweit es eine Rückbindung gibt, etwa durch die konzertierte Aktion, wird ein fiktives Gemeinwohl an die Stelle der politischen Wirkungseinheit im Sinn Hellers gesetzt. Damit laufen Mechanismen der Umverteilung wie Art. is GG leer. Die Eigentumsgarantie soll nun plötzlich, entgegen der früheren Rechtsprechung, auch vor Besteuerungsmaßnahmen schützen und damit eine Umverteilung blockieren. Ein Machtgewinn ergibt sich einzig für die Planungstätigkeit der Regierung. Damit werden jedoch Randgruppen isoliert, Postulate sozialer Homogenität wie die von Heller erhobenen zurückgedrängt. Damit mündete auch dieser Vortrag in die Aktualität der Alternative von Rechtsstaat oder Diktatur.

2. Nach diesen öffentlichen Vorträgen war die gesamte weitere Tagung der konkreten Diskussion unter den Teilnehmern des Symposions gewidmet.

Den Anfang machte eine Bestandsaufnahme über Fakten und Gründe der Rezeption oder Nichtrezeption Hellers, und zwar in der Form einer Spirale. Diese förderte ein frappant schillerndes Bild zutage. So beginnt in Japan die Heller-Rezeption bereits in den dreißiger Jahren im Zeichen des preußischen Einflusses und des monarchischen Scheinkonstitutionalismus, wenn auch neuere Strömungen sich in anderem Sinn auf Heller berufen (Yamagucbi/Aichi, Japan). Auch in Spanien und in Lateinamerika lassen sich ähnlich gebrochene Einflüsse feststellen. Garzón Valdés (Mainz) benannte Theoretiker des Franco-Regimes, der liberalen Opposition dazu und pero- 
nistischer Strömungen in Argentinien, die sich alle auf Heller beriefen. Dieser war zunächst Kronzeuge einer Ablehnung des Positivismus (Kelsen), die Nation und ihre Souveränität betonender Gedanken. Zugleich wurde Heller sogar zum Befürworter naturrechtlicher Positionen stilisiert. Offenbar standen auch Übersetzungsprobleme einer adäquateren Heller-Rezeption entgegen, die erst jetzt beginnt.

Für Italien erscheint der Einflußbereich Hellers zunächst schmal (Bolaffi/Rom, Pasquino/Göttingen). Abgesehen von einigen staatstheoretisch besonders beschlagenen Autoren wie Treves (dazu der Beitrag von Portinaro/Torino) und Mortati, wirkte hier der Faschismus lange Zeit als Filter, der den Einfluß Hellers abwehrte, und selbst auf dem Symposion schien der Schatten Schmitts für die italienischen Gäste bestimmend. Allerdings fasziniert jetzt auch der Vergleich zwischen der Weimarer SPD und dem heutigen PCI: Wie weit darf und muß sich eine Massenpartei mit dem Gedanken nationaler Einheit identifizieren, um regierungsfähig zu werden? Dabei gewinnt für die heutige italienische Fragestellung Heller durch seine Faschismuskritik weiter an Bedeutung.

Weniger kräftige Farbakzente schienen zunächst die weiter nach innen weisenden Drehungen der Spirale aufzuzeigen. Für Großbritannien wies Ellen Kennedy (York), die selbst daran arbeitet, Hellers Werk dort fruchtbar zu machen, auf das Fehlen englischer Übersetzungen und die schon deshalb unterbliebene Rezeption hin. Während zunächst auch die historische Ausgangssituation als "querelle allemande « abgetan werden konnte, zeigt die gegenwärtige Dekadenz von Pluralismus und Parlamentarismus in Großbritannien ähnliche Probleme, die ökonomische Krise und der Versuch, sie zu bewältigen, ähnliche Konfliktsituationen. Über Harold Laski hatte Heller ja in seinen letzten Lebensjahren an der Encyclopedia of Social Sciences mitgearbeitet; als folgenschwer erweist sich, daß es wegen Hellers Tod bei diesen Ansätzen eines Kontakts geblieben ist. - Auch für die deutschsprachigen Staaten sind erhebliche Rezeptionslücken zu konstatieren. So wurde die Bedeutung des gebürtigen Österreichers Heller in seinem Herkunftsland völlig von Kelsen und Max Adler überschattet und erschlossen insofern die für das Symposion gelieferten Beiträge von Kübne (Wien) und Leser (Wien) weitgehend Neuland, das in Verbindung mit der gleich zu referierenden neuen Sicht des Verhältnisses von Heller und Kelsen zu sehen ist. Für die Schweiz scheint eine ähnliche Einschätzung nahe zu liegen, und vor diesem Hintergrund ist die Bedeutung der neuen Arbeit Wasers (Basel) erheblich. $Z$ war bleibt darauf hinzuweisen, daß parallel zu Heller und fast gleichzeitig Dietrich Schindler (Verfassungsrecht und soziale Struktur, 1932) methodisch ähnliche Gedanken wie Heller entwickelte, die das weitere Staatsdenken in der Schweiz beeinflußt haben. Um so auffälliger ist das sozialwissenschaftliche Defizit dieser Richtungen, das erst neuerdings ausgeglichen wird. Auch für die DDR, die immerhin durch Haney (Jena) vertreten war, konstatierte dieser das Ausbleiben jeder Rezeption. Inhaltlich führte er als Teilgrund Hellers Hegel-Mißverständnisse, vor allem hinsichtlich des Wirklichkeitsbegriffs an. Dennoch sah auch er einen gewissen Nachholbedarf für Geschichte und Konstituierung sozialwissenschaftlicher Arbeit in der Staatsrechtswissenschaft der DDR.

Der weitere Gang der Diskussion kreiste vor allem um die Wirkungen Hellers in der Bundesrepublik Deutschland. Auch hier war vorweg die relativ geringe Bedeutung Hellers zu konstatieren (Richter/Hamburg, anders z. B. für Frankfurt Staff). Im übrigen ließ die Diskussion, abgesehen von historisch interessanten Beiträgen zum Klima in der Staatsrechtslehre der Berliner zwanziger Jahre, speziell am KaiserWilhelm-Institut (Brandt/Bonn-Berlin) und zur Einordnung Hellers in einen spezifisch österreichischen $Z$ weig der deutschen Staatsrechtslehre (Müller), vor allem zwei Richtungen erkennen. Die Politikwissenschaftler (insb. Hennig/Kassel, Fijal- 
kowski/Berlin) wiesen auf die Bedeutung Hellers für Carlo Scbmid, M. Friedrich und die Arbeiten des Frankfurter Instituts für Sozialforschung bis hin zu Habermas sowie auf die Umdeutung Hellers durch Abendroth hin, die eine Ablösung von den romantischen, durch die Jugendbewegung geprägten Komponenten voraussetzte. Jedoch seien die jüngeren sozialistischen Autoren der späten Weimarer Republik (Fraenkel, Kirchbeimer, F. Neumann) auf längere Sicht für die heutige Theoriebildung wichtiger. Demgegenüber legten die meisten Vertreter des öffentlichen Rechts (so Schneider/Hannover, Penski/Siegen, Robbers/Karlsruhe, Meinck/Göttingen, Schefold/Bremen u. a.) das Hauptgewicht auf die indirekten Wirkungen Hellers, vor allem in Ergänzung der Smend'schen Integrationslehre. Diese erhielt erst durch Hellers sozialwissenschaftliche Analyse eine konkretere Grundlage, die vom alten Smend auch ausdrücklich akzeptiert wurde und die sich in der Rechtsprechung des Bundesverfassungsgerichts auswirkt. Die Bewertung dieses Methodensynkretismus blieb freilich unterschiedlich. Gegen den Hinweis auf die positive Funktion etwa in der Zeit der Studentenbewegung (Kaiser/Berlin) und für den gegenwärtigen Stand der staatsrechtlichen Methodendiskussion (so die meisten Genannten) setzten andere den Vorwurf des Irrationalismus und der Beliebigkeit der Methode (Meinck, Staff). Am schärfsten argumentierte insofern Abendroth, der die vom Bundesverfassungsgericht unter Berufung auf die sozialwissenschaftlich angereicherte Integrationslehre beanspruchten Interpretationsspielräume kritisierte und betonte, wie stark die deutsche Staatsrechtslehre nach 1945 von den Kontinuitäten der NS-Zeit geprägt war. Wirklich bedeutsam sei nur Hellers Aufdeckung des antagonistischen Charakters des Weimarer Staates und dessen Überbrückung durch die Lehre vom sozialen Rechtsstaat gewesen, die für das Grundgesetz fruchtbar gemacht werden könne. Die Kontroversen ließen allerdings erkennen, daß die eigentliche Frage mehr die nach der Wirkungsgeschichte als nach der konkreten Rezeption individuell zurechenbarer Gedanken war (Rädle/Bonn).

3. Die nächste Gesprächsrunde galt der politischen Verortung der Lehre Hellers zwischen Sozialismus, Faschismus und Nationalismus. Zum Ausgangspunkt nahm Schneider seine Untersuchung des Verhältnisses Hellers zu Radbruch. Von einer engen Zusammenarbeit in Kiel, die auch zu ähnlichen Auffassungen in der Frage des Verhältnisses zur Nation führte, entwickelten sich die Gedankengänge in unterschiedlichen Richtungen, als Radbruch nach Heidelberg ging und in Verbindung zu Anschütz kam, während Heller seine Schriften über "Sozialismus und Nation « und zur Souveränität verfaßte. Die Entfremdung ging so weit, daß Radbruch Heller nach dem Erscheinen von dessen Faschismus-Studie brieflich ironisch fragte, ob er etwa Philofaschist geworden sei, was Heller natürlich, etwas verletzt, zurückwies. Allerdings mochte Radbruch angesichts der positiven Erwähnung der Jugendbewegung, Carl Schmitts und des konkreten Ordnungsdenkens Anlaß zu seiner Frage gesehen haben. Abendroth stimmte dem zu mit dem Hinweis, Heller und Radbruch seien Antikommunisten und insofern - wie viele andere - präfaschistischen Tendenzen zugänglich gewesen; sie hätten die Notwendigkeit autoritärer Elemente auch in der demokratischen Revolution nicht erkannt (ähnlich Haney). Eine andere, klärungsbedürftige Frage sei freilich die nach dem Verhältnis Hellers zu den weit problematischeren Führer-Gedanken Leonhard Nelsons, der ja auch dem Heller entgegengesetzten Hannoveraner Kreis angehörte. Dazu gab Schneider theoriegeschichtliche, Müller einige biographische und wirkungsgeschichtliche Informationen. Albrecht (Hamburg) betonte die analytische Bedeutung der Faschismusschrift.

Indessen blieb gerade diese Frage umstritten. Hennig problematisierte die Konsistenz eines Transformationskonzepts zum Sozialismus in der Form von Hellers Theorie des sozialen Rechtsstaats. Diese sei eher eine autoritär-integrationistische 
Konzeption im Sinn Hegels oder der materiellen Rechtsstaatlichkeit eines Robert von Mohl, klammere aber die Marx'sche Kritik am Gothaer Programm völlig aus und setze, anders als die Arbeiten Kirchheimers und F. Neumanns, den Staat autonom, so daß eine korporatistische Lösung der Klassenfrage denkbar werde. Allerdings habe Heller die lebensphilosophischen Elemente des Faschismus zutreffend kritisiert. Auch Müller räumte das Fehlen eines tragfähigen Transformationskonzepts bei Heller ein, wies aber darauf hin, daß die Staatskritik bei Kirchheimer und Neumann von der Carl Schmitts beeinflußt war, die die Weimarer Verfassung im gegenrevolutionärem Sinn diskreditierte. Die Entscheidung gegen die soziale Transformation sei 1919, als Heller zu schreiben begann, bereits gefallen gewesen, so daß 1931/32 die Option für bürgerliche Demokratie und sozialen Rechtsstaat entgegen diktatorischen Konzepten noch am ehesten aussichtsreich gewesen, die Rückprojektion heutiger Fragestellungen unhistorisch sei. Wichtig waren Wasers und Schneiders Hinweise auf die Entwicklung Hellers. Während in "Sozialismus und Nation« und noch in der Faschismus-Schrift Anklänge an einen Nationalismus mit irrationalen Komponenten vorherrschten, habe Heller spätestens ab 1932, vor allem in der Staatslehre, die Klassenanalyse deutlicher formuliert und das Wesen des Faschismus klarer erfaßt. Auch Lutbardt (Berlin) wies auf die Bedeutung der Verteidigung des Parlamentarismus nach 1930 und die Gefahren einer positiven Bewertung der (linken) Diktatur durch Kirchheimer hin. Historisch sei ein Zusammengehen kommunistischer und sozialistischer Strömungen damals ausgeschlossen, Hellers Entscheidung für den sozialen Rechtsstaat gegen Faschismus, Bolschewismus und bürgerlichen Rechtsstaat daher folgerichtig gewesen. Weitergehend suchten andere Diskussionsredner, insb. Staff und Pasquino, die Heller'sche Theorie als Transformationskonzept zu deuten. Auch Abendroth akzeptierte diese Ansätze, die er für das Grundgesetz fruchtbar zu machen gesucht habe, insistierte freilich für die Weimarer Zeit auf der Alternative Sozialismus oder Barbarei. Gegen letztere habe Heller die Demokratie und damit die soziale Transformation in den konkreten Konflikten nicht hinreichend revolutionär verteidigt.

Konkreter suchte Hennig zeitgeschichtlich Hellers Äußerungen zur sozialen Homogenität (1927) in Verbindung zur Arbeitslosenversicherung, die Äußerungen zum sozialen Rechtsstaat ( 1929) in Verbindung zur sozialdemokratischen Wahlprogrammatik zu bringen. Heller habe aber die Verfassung weniger geschickt als Carl Schmitt zu nutzen gewußt. Seine Interpretation des zweiten Hauptteils der Reichsverfassung sei wirkungslos, die des Prinzips der sozialen Demokratie nicht hinreichend operationalisiert gewesen. Diese Ausführungen wurden durch Bolaffi bei positiverer Würdigung von Hellers Leistung ergänzt, auch durch Parallelen zu Italien. Die Linke habe erst auf den zweiten, ab 1932 auf den ersten Hauptteil der Weimarer Verfassung gesetzt, Carl Schmitt, umgekehrt, erst auf den ersten Hauptteil (Art. 48), dann auf den zweiten (das konkrete Ordnungsdenken). Spekulationen über einen von Schleicher angestrebten »dritten Weg " aus der Sicht von 1932/33 (Brandt) stellte Hentschel (Mainz) eine wirtschaftsgeschichtliche Einordnung von Hellers Theorie entgegen. Von einem sozialdemokratischen Standpunkt aus war eine Rechtsdiktatur ohne Nationalsozialisten ähnlich gefährlich wie diese und daher inakzeptabel. Quintessenz mußte von Hellers Standpunkt aus die Ratlosigkeit sein, die durch seine Staatslehre und deren zeitgenössische Rezeption (bzw. Nicht-Rezeption) hindurchschimmert.

4. Damit war die Diskussion zum Begriff des sozialen Rechtsstaats bereits vorstrukturiert. Meinck relativierte dessen Bedeutung, da es sich nur um die Beschreibung der demokratisch-parlamentarischen Umgestaltung des bürgerlichen Rechtsstaats gehandelt habe, Heller den Bezug zur Antinomie von Rechtsgrundsätzen und posi- 
tivem Recht nicht diskutiert und die Funktion des Begriffs in der Staatslehre nicht weiter entwickelt habe. Abendroth widersprach diesem Befund aus historischer Sicht nicht und gestand zu, daß Sozialstaatlichkeit in jeder die Schwachen unterstützenden Staatstätigkeit, etwa der Sozialgesetzgebung des Kaiserreichs, gesehen werden könne. Aber die Bedeutung von Hellers Begriffsbildung liege darin, daß er den Kompromißcharakter der Weimarer Verfassung benannt habe. Dieser sei zwar im Grundgesetz inhaltlich schwächer, aber als eigentlicher Rechtsgrundsatz verankert, so daß Hellers Beschreibung heute normativ faßbar gemacht werden könne und müsse. Auch Staff bezog den Begriff des sozialen Rechtsstaats in Hellers Konzept der Wandlung des Rechts in der sozialen Wirklichkeit, die die Beliebigkeit der politischen Entscheidung begrenze, ein und betonte die Aktualität dieser Sichtweise. In ähnlichem Sinn sprachen sich Pasquino, Penski und Richter aus, dieser unter Betonung des politischen Formprinzips gegenüber einer bloßen Zusammenfassung einzelner sozialer Rechte, Pasquino mit dem Hinweis auf die wirklichkeitswissenschaftliche, Freyer beeinflussende Methode Hellers, die zwischen Idealismus und historischem Materialismus einen eigenen Stellenwert habe. Wie er sah Waser bei Heller konkret die Verbindung zwischen Analyse des Klassenkampfs und der Fortexistenz der parlamentarischen Demokratie, die beide notwendig seien. Sozialstaatlichkeit bedeute deshalb progressive, praxisbezogene Demokratisierung von unten nach oben. Hennig wandte dagegen ein, Hellers Betonung des Mehrheitsprinzips bleibe einer auch von Konservativen wie Popitz und der Schwerindustrie in Anknüpfung an Theorien des materiellen Rechtsstaats ( $R$. von Mohl) betonten bürgerlichen Sozialpolitik verhaftet. Kirchheimer habe durch die Unterscheidung von Direktionssphäre und Verteilungssphäre die Problematik genauer erfaßt. Schneider zeigte den Zusammenhang zwischen Hellers sozialem Rechtsstaat und Radbruchs Begriff des sozialen Rechts, das mehr Schutz- als Herrschaftscharakter habe, auf, Luthardt den durch die damalige Zeitschrift für soziales Recht und durch F. Neumanns grundrechtstheoretische Schriften bestimmten Rahmen, von dem sich Neumann freilich schon 1934 distanzierte.

5. Von diesem Befund ging die Diskussion zur systematischen Behandlung der Bedeutung der Rechtsgrundsätze bei Heller über. Die Frage erwies sich weitgehend als durch die Auseinandersetzung mit Kelsen geprägt, so daß in erster Linie gefragt wurde, wie beide Autoren die Normativität der Grundsätze begründeten. Paulson (St. Louis/USA) hatte in seinem Beitrag den Wandel des Normativitätsgedankens, vor allem der Bedeutung der hypothetischen Grundnorm, bei Kelsen im Lauf der zwanziger Jahre (gipfelnd in der Schrift von 1928 über die philosophischen Grundlagen der Naturrechtslehre und des Rechtspositivismus) herausgearbeitet. Während die hypothetische Grundnorm zunächst nur logische Voraussetzung der Rechtsordnung gewesen sei, werde sie allmählich im neukantianischen Sinn zur transzendentalen Begründung des Rechts. Wenn Heller Kelsens Grundnorm als bloße Umbenennung des Staatswillens kritisiert habe, so treffe dies Kelsens ursprüngliche Lehre, lasse aber deren Entwicklung außer acht. Hellers Gegnerschaft gegen Kelsen beruhe daher weitgehend auf einem Mißverständnis. In Wirklichkeit sei eine gewisse Konvergenz festzustellen. Diese These wurde durch mehrere Votanten akzeptiert und ergänzt. Schneider wies auf die südwestdeutsche Ausprägung des Neukantianismus (Emil Lask) hin, der einerseits über Radbruch Heller, andererseits auch Kelsen beeinflußt habe, und auf den namentlich die These der Erzeugung der Wirksamkeit und Geltung einer Norm durch deren Befolgung zurückgehe. Auch Bolaffi problematisierte die neukantianisch aufgefaßte Grundnorm, die bei Kelsen zu einer Betonung von Normen und zur Verdrängung des Ausnahmezustands, bei Carl Schmitt jedoch zu einer Politischen Theologie geführt habe, die von Staff als Ideologie kri- 
tisiert wurde. Meinck vermißte bei Heller einen einheitlichen Normativitätsbegriff. Hellers Rechtsgrundsätze würden einerseits als empirische Seinsbegriffe, andererseits als objektive, ethische Idealität verstanden, ähnlich wie Smends Integrationsbegriff und Carl Schmitts Dezision, die ebenfalls nicht beliebig sei. Diesen Positionen sei Kelsens Normativitätsbegriff überlegen. Demgegenüber stellte sich Waser auf Hellers Seite und betonte das Verdienst, das Verhältnis und Sein und Sollen dialektisch zu sehen. Wenn Kelsen die Normativität in das erkennende Subjekt verlagere, so entstehe ein Riß, der durch die gesamte gesellschaftliche Wirklichkeit gehe. Am nachdrücklichsten setzte sich Müller dafür ein, Heller und Kelsen stärker miteinander verbunden zu sehen, um eine juristische Methode, die die geschriebene Verfassung mißachte, zu vermeiden. Insofern behielten Kelsens Vorwürfe gegen Smend, die Integrationslehre sei ein Vorwand zum Verfassungsbruch, ihre Bedeutung, und habe Heller in Kelsen den möglichen Bundesgenossen nicht erkannt. Die logischen Rechtsgrundsätze Hellers seien Kelsens Grundnorm vergleichbar; aber auch bei den ethischen sei eine Annäherung beider Autoren denkbar. Eine neue Sicht des Verhältnisses Hellers zu Kelsen sei daher geboten. Sie wurde, wie die referierten Aussagen zeigen, auf dem Symposion weitgehend strukturiert.

6. Wie die bisherigen Erörterungen gezeigt hatten, bedurfte der Begriff der Entscheidung (Dezision) noch der weiteren Diskussion. Ansatzpunkt war dabei einerseits die Frage, ob die ihre Rechtfertigung in sich selbst tragende Entscheidung im Sinne Carl Schmitts für die Begründung der staatlichen Entscheidungseinheit im Sinn Hellers fruchtbar gemacht werden könne, was insbesondere Scbneider, Albrecht, Staff und Schefold mit dem Hinweis auf den sozialen und rechtlichen Kontext der von Heller beschriebenen Entscheidungssituation verneinten, die bei Schmitt ausgeblendet bleibe; andererseits das Verhältnis der Entscheidung bei Heller zur Konkretisierung des Rechts im Sinn der Reinen Rechtslehre Kelsens, die von Müller und Paulson, in Anlehnung an Einflüsse der Freirechtsschule, ebenfalls als Entscheidungssituation charakterisiert wurde (anders Garzón Valdés). Allerdings hat die Freirechtsschule auch Schmitt beeinflußt (Schefold). Müller akzeptierte dies, wies aber darauf hin, daß Schmitt, von Eugen Ehrlich abweichend, die rechtssoziologischen Grundlagen der Entscheidung nur noch selektiv berücksichtigt habe. Hellers Lehre vom Rechtsbruch sei eine Verarbeitung der Revolutionserfahrungen. Staff betonte ebenfalls, daß die Dezision Schmitts sozial völlig undeterminiert sei, wenn sie auch seinen politischen Zielvorstellungen durchaus habe dienstbar gemacht werden können.

Meinck rückte demgegenüber Heller stärker in die Nähe von Schmitt, da auch dieser eine Legitimation der Entscheidung aus der Akklamation in der identitären Demokratie postuliere und dadurch Ordnungen stiften, das ethische Postulat des inneren Friedens im Sinn von Hobbes verwirklichen wolle. Gewiß sei die Entscheidungssituation, anders bei Kelsen, vom Recht gelöst; aber die Möglichkeit einer Entscheidung gegen das Recht gehöre auch zur Heller'schen Staatslehre. Ähnlich argumentierte Hennig. Schmitts akklamatorische Legitimation sei zwar von Hellers Postulat des Volksbildungsgedankens weit entfernt. Aber die Kritik am Pluralismus und an den intermediären Instanzen, letztlich am quantitativ totalen, schwachen Staat sei als autoritäres politisches Gegenmodell zu Heller höchst fruchtbar gewesen: positiv im analytischen Sinn, wie negativ in der praktischen Politik ab 1933. Auch Penski insistierte auf der realen gesellschaftlichen Verankerung der Schmitt'schen Dezision, sah freilich das Verdienst Hellers ihm und Kelsen gegenüber in der Einbeziehung der Rechtsgrundsätze. Schneider und auch Schefold wiesen darauf hin, daß Schmitt auf Hobbes und Rousseau in totalitäter Intention eklektizistisch Bezug genommen habe, um eine beliebige, freilich bestimmten politischen Zielen dienstbare Entscheidung zu begründen. Hennig sah darin eine geschickte Ausnutzung der staatstheore- 
tischen Quellen wie auch der verfassungsrechtlichen Möglichkeiten, die Schmitt als guten Juristen und Verteidiger realer Interessen des Kapitalismus ausweise. Haney versuchte, die Positionen aus unterschiedlichen Reaktionen auf den sozialen Wandel zum Imperialismus zu erklären, der bei allen drei Autoren den Hintergrund gebildet habe. Das Recht sei als Form der Bewegung von Widersprüchen zu verstehen. Heller habe allerdings den Vorzug, der realen Bedürfnissituation der Menschen am ehesten entsprochen zu haben. Konkreter meinte Schneider, nach Heller könne sich ein Staat, der revolutionäres Recht als sozialer Rechtsstaat verwirkliche, an die Spitze der gesellschaftlichen Entwicklung stellen - was Hennig, freilich nicht in Schneiders Sinn, mit dem Begriff der Diktatur des Proletariats umschrieb. Waser konstatierte insofern eine Entwicklung vom Souveränitätsbuch, in dem Heller die Durchsetzung des Staats auch gegen das Recht betont habe, zur insofern vorsichtigeren, eher auf ein kommunikatives Diskursprinzip ausgerichteten Darstellung in der Staatslehre. Schließlich unterstrich Müller zusammenfassend nochmals die Nähe von Kelsen und Heller in der Frage der Rechtsgrundsätze.

7. Als Schwerpunkt der Abschlußdiskussion, die die noch offenen Fragen - auch aus dem Bereich der Kulturpolitik - einbeziehen sollte, schälte sich vor allem die Überordnung des Staates über die gesellschaftlichen Kräfte heraus. Müller verwies auf Hellers Ablehnung des (insb. spanischen) Anarcho-Sozialismus und den Primat der Politik gegenüber der Wirtschaft, Schneider auf die im Anschluß an Radbruch formulierte Überordnung des Staates über das Recht, Kennedy auf das (auch liberale) Postulat des starken Staats. Dessen Bindungen seien bei Heller (wie bei Schmitt) romantisch verklärt, etwa in den von heute aus antiquierten Schriften zur Arbeiterbildung, während Schmitts politische Theologie die Bindungen des staatlichen Bereichs aus diesem verbanne. Staff hob Hellers Bindungen des Staates, vor allem seinen demokratie-immanenten Kulturbegriff, von Schmitts verfälschter politischer Theologie ab, sah für die Gegenwart jedoch größere Schwierigkeiten, den Interessenpluralismus der Gesellschaft in tragfähige parlamentarische Entscheidungen zu übersetzen.

Kaiser kritisierte die praktische Entscheidungsschwäche Hellers, die sich im Prozeß vor dem Reichsstaatsgerichtshof gegen den Preußenschlag in der defensiven Vorgehensweise Hellers und der SPD gezeigt habe, im Gegensatz zur aggressiv-triumphierenden Position des Schmitt'schen Dezisionismus. Schneider hielt dem die Schwäche der Position Hellers entgegen, da er sich aus der Souveränitätsschrift zu entnehmende Argumente entgegenhalten lassen mußte. Zugleich lehnte Schneider für Heller den Begriff der politischen Romantik ab. Sein Rationalismus führe notwendig zur pluralistisch-parlamentarischen, relativistischen Demokratie, mit Appell-Möglichkeit an das Volk zur Behebung der (ähnlich wie von Schmitt) gesehenen Defizite, wobei allerdings manche Schriften optimistisch und staatsgläubig, andere in der Perspektive düsterer seien. Haney bezeichnete Hellers Staatsbejahung als Reformismus, der die Entfaltung sozialer Energien behindert habe, z. B. im Prozeß Preußen contra Reich.

Luthardt problematisierte die Überordnung des Staates als Monopolisten der physischen Gewalt über die Wirtschaft, wenn dieser Staat in die Wirtschaft intervenieren solle, wie Heller (gegen Schmitt) forderte. Heller habe den Staat statisch in sich ruhend, die Gesellschaft anarchisch gesehen und deshalb den Staat reformistisch einsetzen wollen. Dabei habe er die qualitative Veränderung des Interventionsstaats, wie sie Kirchheimer und Fraenkel analysierten (Bonapartismus-Theorie), übersehen. Entsprechend mußte der Reformismus des sozialen Rechtsstaats an ökonomischen Strukturen scheitern. Albrecht bestritt, daß Hellers Demokratiebegriff relativistisch sei. Er diene den konkret umschriebenen sozialen Zwecken, der Entwicklung zum 
sozialen Rechtsstaat. Das Scheitern dieser Entwicklung sei bei Heller allerdings theoretisch nicht verarbeitet. Diese Aussagen fanden weithin Zustimmung. Insofern zeichnete sich als von mehreren Diskussionsrednern aufgegriffene Formel die des nicht zu Ende gegangenen Weges ab (Haney, Staff).

8. Also eine ermutigend-konstruktive Bilanz, ganz im Sinn von Hellers »verzweifeltem Optimismus «? Das Symposion hat, im Zusammenwirken vieler Wissenschaftler und gewichtiger Beiträge, vie aufgearbeitet, von der noch kaum bekannten internationalen Wirkung Hellers über das bisher wohl vernachlässigte Verhältnis zu Radbruch bis zur in Konturen erkennbaren neuen Sicht des Verhältnisses zu Kelsen. In weiteren Zentralfragen wie der des Verhältnisses zum Dezisionismus und zu Carl Schmitt, der Rolle der Rechtsgrundsätze und der Tragfähigkeit des Prinzips des sozialen Rechtsstaats konnte natürlich kein Konsens, aber doch eine Gegenüberstellung von Meinungen erreicht werden. Sie war fruchtbar und führte manchmal zur Annäherung, mindestens aber zur Verdeutlichung der Standpunkte. Wissenschaftsgeschichtlich wichtig dürfte insofern besonders die Präzisierung von Abendroths Aussagen sein. ${ }^{4} \mathrm{Daß}$ die Fragen der Kulturstaatlichkeit und Arbeiterbildung, zu denen Beiträge von Borinski (Baden-Baden), Richter, Robbers vorlagen, kaum besprochen wurden, ist zwar bedauerlich, aber eine vertretbare Gewichtung.

Auffallender waren andere Lücken. Die Veranstalter haben zwar ausländische Gäste über die Grenzen der Kontinente hinweg, Fachwissenschaftler über den Bereich der Rechtswissenschaft hinaus versammelt und so in eindrücklicher Weise die internationale und interdisziplinäre, besonders auch politikwissenschaftliche Bedeutung Hellers herausgearbeitet. Die deutsche Staatsrechtslehre dagegen war in Grenzen vertreten: abgesehen von ihrem Linksaußen und der einzigen Frau gerade noch durch ein deutsches Mitglied der Staatsrechtslehrer-Vereinigung, sonst durchgängig durch ihr nicht oder noch nicht angehörige Wissenschaftler. Das hat nicht an der Einladungspolitik gelegen. Um so signifikanter ist es für die Fortdauer der Isolierung von Hellers Werk, so sehr es in den Zusammenhang der Entwicklung der deutschen Staatsrechtslehre gehört. Es bleibt zu hoffen, daß auch dieser Widerspruch fruchtbar wird.

Dian Schefold

4 Vgl. Wolfgang Abendroth, Ein Leben in der Arbeiterbewegung, Frankfurt 1976, S. 145 f., 2 I 3 f. 